\title{
A FUNCTIONAL INEQUALITY ON THE BOUNDARY OF STATIC MANIFOLDS
}

\author{
KWOK-KUN KWONG\# AND PENGZI MIAO*
}

\begin{abstract}
On the boundary of a compact Riemannian manifold $(\Omega, g)$ whose metric $g$ is static, we establish a functional inequality involving the static potential of $(\Omega, g)$, the second fundamental form and the mean curvature of the boundary $\partial \Omega$ respectively.
\end{abstract}

\section{INTRODUCTION AND STATEMENT OF RESULTS}

The research in this paper is largely motivated by the following result concerning a functional inequality on the boundary of bounded domains in the Euclidean space $\mathbb{R}^{n}$, proved in [11, Corollary 3.1].

Theorem 1 ([11]). Let $\Omega \subset \mathbb{R}^{n}$ be a bounded domain with smooth boundary $\Sigma$. Let $H$ and $\mathbb{I I}$ be the mean curvature and the second fundamental form of $\Sigma$ with respect to the outward normal respectively. If $H>0$, then

$$
\int_{\Sigma}\left[\frac{\left(\Delta_{\Sigma} \eta\right)^{2}}{H}-\mathbb{I} \mathbb{I}\left(\nabla_{\Sigma} \eta, \nabla_{\Sigma} \eta\right)\right] d \sigma \geq 0
$$

for any smooth function $\eta$ on $\Sigma$. Here $\nabla_{\Sigma}, \Delta_{\Sigma}$ denote the gradient, the Laplacian on $\Sigma$ respectively, and $d \sigma$ is the volume form on $\Sigma$. Moreover, equality in (1.1) holds for some $\eta$ if and only if $\eta=a_{0}+\sum_{i=1}^{n} a_{i} x_{i}$ for some constants $a_{0}, a_{1}, \ldots, a_{n}$. Here $\left\{x_{1}, \ldots, x_{n}\right\}$ are the standard coordinate functions on $\mathbb{R}^{n}$.

When $n=3$ and $\Sigma$ is convex, it is known (11]) that the functional on the left side of (1.1) represents the second variation along $\eta$ of the Wang-Yau quasi-local energy ([16, 17]) at the 2 -surface $\Sigma$, lying in the time-symmetric slice $\mathbb{R}^{3}=\{t=0\}$, in the Minkowski spacetime $\mathbb{R}^{3,1}$. Thus, (1.1) can be relativistically interpreted as the stability inequality of the Wang-Yau energy at $\Sigma$. The general case of such a stability

\footnotetext{
\#Research partially supported by Ministry of Science and Technology in Taiwan under grant MOST103-2115-M-006-016-MY3.

*Research partially supported by Simons Foundation Collaboration Grant for Mathematicians \#281105.
} 
inequality is implied by results in [6, 17] for a closed, embedded, spacelike 2-surface in $\mathbb{R}^{3,1}$ that projects to a convex 2-surface along some timelike direction.

In this paper, adopting a Riemannian geometry point of view, we generalize Theorem 1 to hypersurfaces that are boundaries of bounded domains in a simply connected space form. More generally, we give an analogue of (1.1) on the boundary of compact Riemannian manifolds whose metrics are static (see Definition 1).

First, we fix some notations. Given a constant $\kappa>0$, let $\mathbb{H}^{n}(\kappa)$ and $\mathbb{S}_{+}^{n}(\kappa)$ denote an $n$-dimensional hyperbolic space of constant sectional curvature $-\kappa$ and an $n$-dimensional open hemisphere of constant sectional curvature $\kappa$ respectively.

Theorem 2. Suppose $(M, g)$ is one of $\mathbb{R}^{n}, \mathbb{H}^{n}(\kappa)$ and $\mathbb{S}_{+}^{n}(\kappa)$. Let $V$ be the positive function on $M$ given by

$$
V= \begin{cases}1, & \text { if }(M, g)=\mathbb{R}^{n}, \\ \cosh \sqrt{\kappa} r, & \text { if }(M, g)=\mathbb{H}^{n}(\kappa), \\ \cos \sqrt{\kappa} r, & \text { if }(M, g)=\mathbb{S}_{+}^{n}(\kappa),\end{cases}
$$

where $r$ is the distance function from a fixed point $p$ on $(M, g)$. When $(M, g)=\mathbb{S}_{+}^{n}(\kappa), p$ is chosen to be the center of $\mathbb{S}_{+}^{n}(\kappa)$ so that $V>0$ on $M$. Given a bounded domain $\Omega \subset M$ with smooth boundary $\Sigma$, let $H$ and $\mathbb{I I}$ be the mean curvature and the second fundamental form of $\Sigma$ respectively. If $H>0$, then for any smooth function $\eta$ on $\Sigma$,

$$
\begin{aligned}
& \int_{\Sigma} V\left[\frac{\left[\Delta_{\Sigma} \eta+(n-1) k \eta\right]^{2}}{H}-\mathbb{I I}\left(\nabla_{\Sigma} \eta, \nabla_{\Sigma} \eta\right)\right] d \sigma \\
\geq & \int_{\Sigma} \frac{\partial V}{\partial \nu}\left[\left|\nabla_{\Sigma} \eta\right|^{2}-(n-1) k \eta^{2}\right] d \sigma .
\end{aligned}
$$

Here $k=0$ or $\pm \kappa$ is the sectional curvature of $(M, g)$. Moreover, equality in (1.3) holds if and only if $\eta$ is the restriction of a function

$$
u= \begin{cases}a_{0}+\sum_{i=1}^{n} a_{i} x_{i}, & \text { if }(M, g)=\mathbb{R}^{n}, \\ a_{0} t+\sum_{i=1}^{n} a_{i} x_{i}, & \text { if }(M, g)=\mathbb{H}^{n}(\kappa), \\ a_{0} x_{0}+\sum_{i=1}^{n} a_{i} x_{i}, & \text { if }(M, g)=\mathbb{S}_{+}^{n}(\kappa) .\end{cases}
$$

Here $a_{0}, \ldots, a_{n}$ are arbitrary constants, $\mathbb{H}^{n}(\kappa)$ is identified with

$$
\left\{\left(t, x_{1}, \ldots, x_{n}\right) \in \mathbb{R}^{n, 1} \mid-t^{2}+\sum_{i=1}^{n} x_{i}^{2}=-\frac{1}{\kappa}, t>0\right\}
$$


in the $(n+1)$-dimensional Minkowski space $\mathbb{R}^{n, 1}$ and $\mathbb{S}_{+}^{n}(\kappa)$ is identified with

$$
\left\{\left(x_{0}, x_{1}, \ldots, x_{n}\right) \in \mathbb{R}^{n+1} \mid \sum_{i=0}^{n} x_{i}^{2}=\frac{1}{\kappa}, x_{0}>0\right\}
$$

in the $(n+1)$-dimensional Euclidean space $\mathbb{R}^{n+1}$.

The standard metrics on $\mathbb{R}^{n}, \mathbb{H}^{n}(\kappa), \mathbb{S}_{+}^{n}(\kappa)$ are all examples of static metrics which admit a positive static potential. We recall the following definition from [9]:

Definition 1 ([9]). A Riemannian metric $g$ on a manifold $M$ is called static if the linearized scalar curvature map at $g$ has a nontrivial cokernel, i.e. if there exists a nontrivial function $f$ on $M$ such that

$$
-(\Delta f) g+\nabla^{2} f-f \text { Ric }=0 .
$$

Here $\nabla^{2}, \Delta$ and Ric denote the Hessian, the Laplacian and the Ricci curvature of $g$ respectively.

On a connected $(M, g)$ of dimension $n$, the space of functions $f$ satisfying (1.5) has dimension at most $n+1$ (cf. [9, Corollary 2.4]). When $g$ is static on $M$, a nontrivial solution $f$ to $(1.5)$ is called a static potential of $(M, g)$.

It is known that a static metric necessarily has constant scalar curvature (cf. [9, Proposition 2.3]). Indeed, direct calculation shows that $(M, g)$ is static with a positive static potential $f$ if and only if the Lorentz warped product $\bar{g}=-f^{2} d t^{2}+g$ satisfies $\operatorname{Ric}(\bar{g})=\frac{R}{n-1} \bar{g}$ where $R$ is the scalar curvature of $g$ (cf. [9, Proposition 2.7]). This interpretation explains why static metrics have been widely studied in the field of mathematical relativity (see e.g. [3, 1, 9, 17, 2, 8, 10, ).

Our next theorem generalizes Theorem 10 to the boundary of a compact Riemannian manifold whose metric is static.

Theorem 3. Suppose $g$ is a static metric on an $n$-dimensional compact manifold $\Omega$ with boundary $\Sigma$ and $V$ is a positive static potential on $(\Omega, g)$. Let $H, \mathbb{I I}$ be the mean curvature, the second fundamental form of $\Sigma$ in $(\Omega, g)$ respectively. If $H>0$, then

$$
\begin{aligned}
& \int_{\Sigma} V\left[\frac{\left[\Delta_{\Sigma} \eta+(n-1) k \eta\right]^{2}}{H}-\mathbb{I I}\left(\nabla_{\Sigma} \eta, \nabla_{\Sigma} \eta\right)\right] d \sigma \\
\geq & \int_{\Sigma} \frac{\partial V}{\partial \nu}\left[\left|\nabla_{\Sigma} \eta\right|^{2}-(n-1) k \eta^{2}\right] d \sigma
\end{aligned}
$$

for any function $\eta$ on $\Sigma$. Here $k \leq 0$ is a nonpositive constant satisfying Ric $\geq(n-1) \mathrm{kg}$. Moreover, equality holds only if 
(i) $k=0$ and $\eta$ is the boundary value of a function $u$ on $(\Omega, g)$ satisfying $\nabla^{2} u=0$.

or

(ii) $k<0, g$ is Einstein, i.e. Ric $=(n-1) k g$, and $\eta$ is the boundary value of a function $u$ on $(\Omega, g)$ satisfying $\nabla^{2} u+k u g=0$.

In Theorem 3, the fact that $k$ is taken as a nonpositive lower bound of the Ricci curvature of $g$ is restricted by the method of our proof (cf. Remark 2.2). Thus, if $g$ has positive Ricci curvature, (1.6) is always a strict inequality. However, in this case, if in addition that $g$ is Einstein, then $k$ can be chosen to be positive and (1.6) is sharp (cf. Remark 2.3).

If the metric $g$ is not static, we also give an inequality similar to that in Theorem 3 but under more stringent assumptions on the boundary and the interior curvature (see Theorem 4).

\section{PROOF OF THEOREMS 2 AND 3}

Theorem [1 was derived in [1] as an application of Reilly's formula [13]. (A different generalization of Theorem 1 was given in [12], again by making use of Reilly's formula.) To prove Theorem 2 and 3, we make use of the following weighted Reilly's formula, recently derived by Qiu and Xia in [14, Theorem 1.1].

Proposition 1 ([14]). Let $(\Omega, g)$ be an $n$-dimensional, compact Riemannian manifold with boundary $\Sigma$. Given two functions $f, V$ on $\Omega$ and a constant $K$, one has

$$
\begin{aligned}
& \int_{\Omega} V\left[(\Delta f+K n f)^{2}-\left|\nabla^{2} f+K f g\right|^{2}\right] d v \\
= & \int_{\Omega}\left[\nabla^{2} V-(\Delta V) g-2(n-1) K V g+V \operatorname{Ric}\right](\nabla f, \nabla f) d v \\
& +(n-1) K \int_{\Omega}(\Delta V+n K V) f^{2} d v+\int_{\Sigma} \frac{\partial V}{\partial \nu}\left[\left|\nabla_{\Sigma} f\right|^{2}-(n-1) K f^{2}\right] d \sigma \\
& +\int_{\Sigma} V\left[2\left(\frac{\partial f}{\partial \nu}\right) \Delta_{\Sigma} f+H\left(\frac{\partial f}{\partial \nu}\right)^{2}+\mathbb{I I}\left(\nabla_{\Sigma} f, \nabla_{\Sigma} f\right)+2(n-1) K\left(\frac{\partial f}{\partial \nu}\right) f\right] d \sigma .
\end{aligned}
$$

For readers' convenience, we include a proof of (2.1) below.

Proof. Direct calculation gives

$$
\frac{1}{2} \Delta\left(V|\nabla f|^{2}\right)=\frac{1}{2}(\Delta V)|\nabla f|^{2}+\frac{1}{2} V \Delta|\nabla f|^{2}+\left\langle\nabla V, \nabla|\nabla f|^{2}\right\rangle .
$$


The integral of $\left\langle\nabla V, \nabla|\nabla f|^{2}\right\rangle$ can be written as

$$
\begin{aligned}
& \int_{\Omega}\left\langle\nabla V, \nabla|\nabla f|^{2}\right\rangle d v \\
= & \frac{3}{2} \int_{\Omega}\left\langle\nabla V, \nabla|\nabla f|^{2}\right\rangle-\int_{\Omega} \nabla^{2} f(\nabla V, \nabla f) d v \\
= & -\frac{3}{2} \int_{\Omega}(\Delta V)|\nabla f|^{2} d v+\frac{3}{2} \int_{\Sigma} \frac{\partial V}{\partial \nu}|\nabla f|^{2} d \sigma-\int_{\Sigma}\langle\nabla V, \nabla f\rangle \frac{\partial f}{\partial \nu} d \sigma \\
& +\int_{\Omega} \nabla^{2} V(\nabla f, \nabla f) d v+\int_{\Omega}\langle\nabla V, \nabla f\rangle \Delta f d v .
\end{aligned}
$$

It follows from (2.2), (2.3) and the Bochner formula that

$$
\begin{aligned}
& \frac{1}{2} \int_{\Sigma} \frac{\partial}{\partial \nu}\left(V|\nabla f|^{2}\right) d \sigma-\int_{\Omega} V\left[\left|\nabla^{2} f\right|^{2}+\operatorname{Ric}(\nabla f, \nabla f)+\langle\nabla \Delta f, \nabla f\rangle\right] d v \\
= & -\int_{\Omega}(\Delta V)|\nabla f|^{2}+\frac{3}{2} \int_{\Sigma} \frac{\partial V}{\partial \nu}|\nabla f|^{2}-\int_{\Sigma}\langle\nabla V, \nabla f\rangle \frac{\partial f}{\partial \nu} d \sigma \\
& +\int_{\Omega} \nabla^{2} V(\nabla f, \nabla f) d v+\int_{\Omega}\langle\nabla V, \nabla f\rangle \Delta f d v .
\end{aligned}
$$

Using the fact

$$
\begin{aligned}
\frac{1}{2} \frac{\partial}{\partial \nu}|\nabla f|^{2}= & \left\langle\nabla_{\Sigma} f, \nabla_{\Sigma}\left(\frac{\partial f}{\partial \nu}\right)\right\rangle-\mathbb{I} \mathbb{I}\left(\nabla_{\Sigma} f, \nabla_{\Sigma} f\right) \\
& +\frac{\partial f}{\partial \nu}\left(\Delta f-\Delta_{\Sigma} f-H \frac{\partial f}{\partial \nu}\right)
\end{aligned}
$$

and

$$
\begin{aligned}
\int_{\Omega} V\langle\nabla \Delta f, \nabla f\rangle d v & =-\int_{\Omega} V(\Delta f)^{2} d v-\int_{\Omega}\langle\nabla V, \nabla f\rangle \Delta f d v \\
& +\int_{\Sigma} V(\Delta f) \frac{\partial f}{\partial \nu} d \sigma,
\end{aligned}
$$

we have

$$
\begin{aligned}
& \int_{\Sigma} V\left[-\mathbb{I I}\left(\nabla_{\Sigma} f, \nabla_{\Sigma} f\right)+\frac{\partial f}{\partial \nu}\left(-2 \Delta_{\Sigma} f-H \frac{\partial f}{\partial \nu}\right)\right] d \sigma-\int_{\Sigma} \frac{\partial V}{\partial \nu}\left|\nabla_{\Sigma} f\right|^{2} d \sigma \\
= & \int_{\Omega} V\left[\left|\nabla^{2} f\right|^{2}-(\Delta f)^{2}\right]+\left[V \operatorname{Ric}-(\Delta V) g+\nabla^{2} V\right](\nabla f, \nabla f) d v,
\end{aligned}
$$

where we also made the use of

$$
\int_{\Sigma} V\left\langle\nabla_{\Sigma} f, \nabla_{\Sigma}\left(\frac{\partial f}{\partial \nu}\right)\right\rangle+\left\langle\nabla_{\Sigma} V, \nabla_{\Sigma} f\right\rangle \frac{\partial f}{\partial \nu} d \sigma=-\int_{\Sigma} V\left(\Delta_{\Sigma} f\right) \frac{\partial f}{\partial \nu} d \sigma
$$


and $|\nabla f|^{2}=\left(\frac{\partial f}{\partial \nu}\right)^{2}+\left|\nabla_{\Sigma} f\right|^{2}$ along $\Sigma$. Now (2.1) follows from (2.6) and the fact

$$
\begin{aligned}
& \int_{\Omega} V\left[\left|\nabla^{2} f\right|^{2}-(\Delta f)^{2}\right] d v \\
= & \int_{\Omega} V\left[\left|\nabla^{2} f+K f g\right|^{2}-(\Delta f+n K f)^{2}\right] d v+(n-1) K \int_{\Omega} n K V f^{2} d v \\
& +(n-1) K\left[\int_{\Sigma}\left(2 V f \frac{\partial f}{\partial \nu}-f^{2} \frac{\partial V}{\partial \nu}\right) d \sigma+\left(\int_{\Omega}(\Delta V) f^{2}-2 V|\nabla f|^{2}\right) d v\right] .
\end{aligned}
$$

This completes the proof.

Remark 2.1. Formula (2.1) reduces to Reilly's formula ([13, equation(14)]) when $V=1$ and $K=0$.

Motivated by equation (1.5) in Definition 1 of static metrics, we can rewrite formula (2.1) as

$$
\begin{aligned}
& \int_{\Omega} V\left[(\Delta f+K n f)^{2}-\left|\nabla^{2} f+K f g\right|^{2}\right] d v \\
= & \int_{\Omega}\left[\nabla^{2} V-(\Delta V) g-V \mathrm{Ric}\right](\nabla f, \nabla f) d v+2 \int_{\Omega} V[\operatorname{Ric}-(n-1) K g](\nabla f, \nabla f) d v \\
& +(n-1) K \int_{\Omega}(\Delta V+n K V) f^{2} d v+\int_{\Sigma} \frac{\partial V}{\partial \nu}\left[\left|\nabla_{\Sigma} f\right|^{2}-(n-1) K f^{2}\right] d \sigma \\
& +\int_{\Sigma} V\left[2\left(\frac{\partial f}{\partial \nu}\right) \Delta_{\Sigma} f+H\left(\frac{\partial f}{\partial \nu}\right)^{2}+\mathbb{I} \mathbb{I}\left(\nabla_{\Sigma} f, \nabla_{\Sigma} f\right)+2(n-1) K\left(\frac{\partial f}{\partial \nu}\right) f\right] d \sigma .
\end{aligned}
$$

It is the second line in (2.7) that prompts one to apply Proposition 1 to domains in a static manifold.

Proof of Theorem 3. As $k \leq 0$, given any nontrivial $\eta$ on $\Sigma$, there exists a unique solution $u$ to

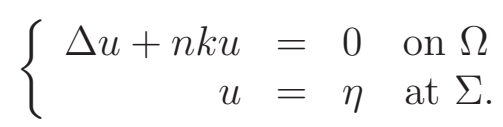

On the other hand, taking trace of (1.5) gives

$$
\Delta V+\frac{R}{n-1} V=0
$$

where $R$ is the scalar curvature of $g$ (which is a constant). Plug this $V$, together with $f=u$ and $K=k$ in (2.1), using (1.5), (2.7) and (2.9), 
we have

$$
\begin{aligned}
& -\int_{\Omega} V\left|\nabla^{2} u+k u g\right|^{2} d v \\
= & 2 \int_{\Omega} V[\operatorname{Ric}-(n-1) k g](\nabla u, \nabla u) d v+k[n(n-1) k-R] \int_{\Omega} V u^{2} d v \\
& +\int_{\Sigma} \frac{\partial V}{\partial \nu}\left[\left|\nabla_{\Sigma} \eta\right|^{2}-(n-1) k \eta^{2}\right] d \sigma \\
& +\int_{\Sigma} V\left[2\left(\frac{\partial u}{\partial \nu}\right) \Delta_{\Sigma} \eta+H\left(\frac{\partial u}{\partial \nu}\right)^{2}+\mathbb{I}\left(\nabla_{\Sigma} \eta, \nabla_{\Sigma} \eta\right)+2(n-1) k\left(\frac{\partial u}{\partial \nu}\right) \eta\right] d \sigma .
\end{aligned}
$$

Since $V>0$, Ric $\geq(n-1) k g, R \geq n(n-1) k$ and $k \leq 0$, (2.10) implies

$$
\begin{aligned}
& \int_{\Sigma} V\left\{\frac{\left[\Delta_{\Sigma} \eta+(n-1) k \eta\right]^{2}}{H}-\mathbb{I I}\left(\nabla_{\Sigma} \eta, \nabla_{\Sigma} \eta\right)\right\} d \sigma \\
\geq & \int_{\Omega} V\left|\nabla^{2} u+k u g\right|^{2} d v+\int_{\Sigma} \frac{\partial V}{\partial \nu}\left[\left|\nabla_{\Sigma} \eta\right|^{2}-(n-1) K \eta^{2}\right] d \sigma \\
& +\int_{\Sigma} V\left[\sqrt{H}\left(\frac{\partial u}{\partial \nu}\right)+\frac{\Delta_{\Sigma} \eta+(n-1) k \eta}{\sqrt{H}}\right]^{2} d \sigma .
\end{aligned}
$$

It follows from (2.11) that

$$
\begin{aligned}
& \int_{\Sigma} V\left\{\frac{\left[\Delta_{\Sigma} \eta+(n-1) k \eta\right]^{2}}{H}-\mathbb{I}\left(\nabla_{\Sigma} \eta, \nabla_{\Sigma} \eta\right)\right\} d \sigma \\
\geq & \int_{\Sigma} \frac{\partial V}{\partial \nu}\left[\left|\nabla_{\Sigma} \eta\right|^{2}-(n-1) K \eta^{2}\right] d \sigma .
\end{aligned}
$$

Moreover, by (2.10), equality in (2.12) holds only if

$$
\begin{aligned}
k[n(n-1) k-R] & =0, \\
\nabla^{2} u+k u g & =0, \\
H\left(\frac{\partial u}{\partial \nu}\right)+\Delta_{\Sigma} \eta+(n-1) k \eta & =0 .
\end{aligned}
$$

Condition (2.13) implies either $k=0$ or $R=n(n-1) k$. In the later case, it follows from Ric $\geq(n-1) k g$ that Ric $=(n-1) k g$, i.e. $g$ is Einstein. We also note that (2.15) in fact follows from (2.14). This is because, if (2.14) holds, then at $\Sigma$,

$$
\Delta u=\Delta_{\Sigma} u+H \frac{\partial u}{\partial \nu}+\nabla^{2} u(\nu, \nu)=\Delta_{\Sigma} u+H \frac{\partial u}{\partial \nu}-k u
$$

which implies (2.15) since $\Delta u=-n k u$. This proves Theorem 3 , 
Remark 2.2. In the above proof, the assumption $k \leq 0$ is essentially used in only one place, i.e. to ensure

$$
k[n(n-1) k-R] \geq 0 .
$$

The other use of $k \leq 0$ in the construction of $u$ is not essential because, by another theorem of Reilly ([13, Theorem 4]), one can still solve (2.8) in the case of $k>0$, provided $(\Omega, g)$ is not isometric to $\mathbb{S}_{+}^{n}(k)$.

Remark 2.3. If Ric $=(n-1) \mathrm{kg}$, then

$$
k[n(n-1) k-R]=0
$$

regardless of the sign of $k$. Therefore, the above proof also shows that inequality (1.6) still holds if the assumption "Ric $\geq(n-1) \mathrm{kg}$ and $k \leq 0$ " is replaced by that $g$ is Einstein. In this case, equality holds if and only if $\eta$ is the boundary value of some function $u$ that satisfies $\nabla^{2} u+k u g=0$ on $(\Omega, g)$.

Theorem 2 now follows from Theorem 3 and Remark 2.3,

Proof of Theorem 2. Each positive function $V$ in (1.2) is a solution to (1.5) when $(M, g)=\mathbb{R}^{n}, \mathbb{H}^{n}(\kappa)$ or $\mathbb{S}_{+}^{n}(\kappa)$. Hence, inequality (1.3) follows from (1.6) in Theorem 3 and Remark 2.3.

Suppose the equality in (1.3) holds from a nontrivial $\eta$. By Theorem 3 and Remark 2.3, $\eta$ is the boundary value of a function $u$ on $(\Omega, g)$ satisfying

$$
\nabla^{2} u+k u g=0 .
$$

Since the standard metric $g$ on $\mathbb{R}^{n}, \mathbb{H}^{n}(\kappa)$ and $\mathbb{S}_{+}^{n}(\kappa)$ is also Einstein, the static equation (1.5) is equivalent to

$$
\nabla^{2} f+k f g=0
$$

Therefore, $u$ is the restriction of a static potential of $(M, g)$ to $(\Omega, g)$. Theorem 2 now follows from the fact that the space of solutions to (1.5) on $(M, g)$ is spanned by

$$
\begin{aligned}
\left\{1, x_{1}, \ldots, x_{n}\right\}, & \text { when }(M, g)=\mathbb{R}^{n} \\
\left\{t, x_{1}, \ldots, x_{n}\right\}, & \text { when }(M, g)=\mathbb{H}^{n}(\kappa) \\
\left\{x_{0}, x_{1}, \ldots, x_{n}\right\}, & \text { when }(M, g)=\mathbb{S}_{+}^{n}(\kappa) .
\end{aligned}
$$

Remark 2.4. By [4] (p. 192-194) (cf. [15] Theorem 2 for a related result), it is known that if $(\Omega, g)$ possesses a function $u$ with $\nabla^{2} u=$ $-k u g$, then $g$ is locally a warped product metric in the sense that there exists a Riemannian manifold $\left(N^{n-1}, g_{N}\right)$ such that $g$ can be locally expressed as $d r^{2}+s(r)^{2} g_{N}$ where $s(r)$ is a function on an interval $I$. 
In fact, their argument (which is local) shows that $u$ can be expressed as a function of $r$ and $u(r)$ satisfies the linear ODE $u^{\prime \prime}=-k u$, and that $s(r)=u^{\prime}(r)$. Also, $s=u^{\prime}$ and $g_{N}$ are unique up to multiplicative constants. Once these have been fixed, $u$ is determined by an additive constant. For example, when $k=0, g$ is locally a product metric $d r^{2}+g_{N}$.

\section{A SIMILAR INEQUALITY}

When the metric is not static, there is an inequality similar to that in Theorem 3 but under more stringent conditions on the boundary and the interior curvature.

For a compact Riemannian manifold $\Omega$ with boundary $\Sigma$, we say it is star-shaped with respect to an interior point $p \in \Omega$ if every point in $\Omega$ can be joined by a minimal geodesic starting from $p$.

Theorem 4. Let $(\Omega, g)$ be an $n$-dimensional compact Riemannian manifold with boundary $\Sigma$. Suppose $\Sigma$ has positive mean curvature and is star-shaped with respect to an interior point $p \in \Omega$. Let $\kappa>0$ be a constant such that $-\kappa$ is a lower bound of the sectional curvature of $g$. Let $r=d(p, \cdot)$ and $V=\cosh \sqrt{\kappa} r$. Here $d(\cdot, \cdot)$ denotes the distance function on $(\Omega, g)$. Then for any function $\eta$ on $\Sigma$,

$$
\begin{aligned}
& \int_{\Sigma} V\left[\frac{\left[\Delta_{\Sigma} \eta-(n-1) \kappa \eta\right]^{2}}{H}-\mathbb{I I}\left(\nabla_{\Sigma} \eta, \nabla_{\Sigma} \eta\right)\right] d \sigma \\
\geq & \int_{\Sigma} \frac{\partial V}{\partial \nu}\left[\left|\nabla_{\Sigma} \eta\right|^{2}+(n-1) \kappa \eta^{2}\right] d \sigma .
\end{aligned}
$$

Moreover, the equality holds only if $\Omega$ has constant curvature $-\kappa$. Here $H, \mathbb{I I}$ are the mean curvature and the second fundamental form of $\Sigma$ respectively.

Proof. By Hessian comparison, we have

$$
\nabla^{2} r \leq \sqrt{\kappa} \operatorname{coth}(\sqrt{\kappa} r)\left(g-d r^{2}\right)
$$

This implies

$$
\nabla^{2} V=\sqrt{\kappa} \sinh (\sqrt{\kappa} r) \nabla^{2} r+\kappa \cosh (\sqrt{\kappa} r) d r^{2} \leq \kappa \cosh (\sqrt{\kappa} r) g=\kappa V g .
$$

By diagonalizing $\nabla^{2} V$, we see that

$$
\Delta V g-\nabla^{2} V \leq(n-1) \kappa V g
$$

and $\Delta V \leq n \kappa V$. This implies that, for any function $u$ on $\Omega$,

$$
\int_{\Omega}\left(V \operatorname{Ric}+2(n-1) \kappa V g+\nabla^{2} V-\Delta V g\right)(\nabla u, \nabla u) d v \geq 0 .
$$


The proof then proceeds as in Theorem 3 ,

If the equality case holds, then as in the argument of Theorem 3 , we have $R=-n(n-1) \kappa$, which implies $\Omega$ has constant curvature $-\kappa$ as we assume its curvature $\geq-\kappa$.

Acknowledgements. PM would like to thank Shanghai Center for Mathematical Sciences for its gracious hospitality, during which part of the work on this paper was carried out. Both authors would like to thank the anonymous referee for the very useful comments and suggestions.

After this paper was submitted, we learned that an inequality that is analogous to (1.3) in Theorem 2 was established by Chen, Wang and Yau $([5])$ in the study of quasi-local energy for spacetimes with a cosmological constant. We want to thank Professors Po-Ning Chen and Mu-Tao Wang for helpful discussions concerning (1.3).

\section{REFERENCES}

[1] Anderson, M. T., On the structure of solutions to the static vacuum Einstein equations, Ann. Henri Poincaré 1 (2000), no. 6, 995-1042.

[2] Beig, R. and Schoen, R., On static n-body configurations in relativity, Classical Quantum Gravity 26 (2009), no. 7, 075014, 7 pp.

[3] Bunting, G. L. and Masood-ul-Alam, A.K.M., Nonexistence of multiple black holes in asymptotically euclidean static vacuum space-time, Gen. Relativity Gravitation 19 (1987), no. 2, 147-154.

[4] Cheeger, J. and Colding T. H., Lower bounds on Ricci curvature and the almost rigidity of warped products, Ann. Math. 144 (1996), no. 1, 189-237.

[5] Chen, P.-N., Quasi-local energy for spacetimes with a cosmology constant, presentation at the Conference in Mathematical General Relativity, TSIMF, Sanya, January 2016.

[6] Chen, P.-N., Wang, M.-T. and Yau, S.-T., Minimizing properties of critical points of quasi-local energy, Comm. Math. Phys. 329 (2014), no. 3, 919-935.

[7] Chruściel, P. T., The classification of static vacuum spacetimes containing an asymptotically flat spacelike hypersurface with compact interior, Classical Quantum Gravity 16 (1999), no. 3, 661-687.

[8] Chruściel, P. T. and Galloway, G. J., Uniqueness of static black holes without analyticity, Classical Quantum Gravity 27 (2010), no. 15, 152001, 6 pp.

[9] Corvino, J., Scalar curvature deformation and a gluing construction for the Einstein constraint equations, Comm. Math. Phys. 214 (2000), 137-189.

[10] Mars, M. and Reiris, M., Global and uniqueness properties of stationary and static spacetimes with outer trapped surfaces, Comm. Math. Phys. 322 (2013), no. 2, 633-666.

[11] Miao, P., Tam, L.-F. and Xie, N.-Q., Critical points of Wang-Yau quasi-local energy, Ann. Henri Poincaré 12 (2011), no. 5, 987-1017.

[12] Miao, P. and Wang, X., Boundary effect of Ricci curvature, arXiv:1408.2711, to appear in J. Diff. Geom. 
[13] Reilly, R. C., Applications of the Hessian operator in a Riemannian manifold, Indiana Univ. Math. J. 26 (1977), 459-472.

[14] Qiu, G.-H. and Xia, C., A generalization of Reilly's formula and its applications to a new Heintze-Karcher type inequality, Int. Math. Res. Notices 17 (2015), 7608-7619.

[15] Tashiro, Y., Complete Riemannian manifolds and some vector fields, Trans. Amer. Math. Soc. 117 (1965), 251-275.

[16] Wang, M.-T. and Yau, S.-T., Quasilocal mass in general relativity, Phys. Rev. Lett. 102 (2009), 021101.

[17] Wang, M.-T. and Yau, S.-T., Isometric embeddings into the Minkowski space and new quasi-local mass, Comm. Math. Phys. 288(3): 919-942, 2009.

(Kwok-Kun Kwong) Department of Mathematics, National Cheng Kung University, TAINAN City 70101, TAIWAN

E-mail address: kwong@mail.ncku.edu.tw

(Pengzi Miao) Department of Mathematics, University of Miami, Coral Gables, FL 33146, USA.

E-mail address: pengzim@math.miami.edu 